\title{
Der Reuchlin-Gedenkstein aus dem Kreuzgang der Stuttgarter Dominikanerkirche
}

\author{
Von Karl Halbauer
}

Der Inschriftenstein des humanistischen Gelehrten Johannes Reuchlin (14551522), der sich heute in der Stuttgarter Leonhardskirche befindet, ist eine hochrechteckige Platte mit drei vertieften Schriftfeldern ${ }^{1}$ (Abb.1). Über dem Bogen des Hauptfeldes trennt ein kurzes tauartig gewundenes Band die beiden Eckzwickel voneinander. (Man könnte den Eindruck gewinnen, der Bogen hinge an einem Strick $)^{2}$. Die lateinische Inschrift des Hauptfeldes ${ }^{3}$ lautet übersetzt: „Im Jahr Christi 1501 (hat) sich und den Reuchlinschen Nachkommen Johannes Reuchlin von Pforzheim (dieses Denkmal gewidmet).“ Im linken Zwickel steht auf Hebräisch: „Ewiges Leben“4, im rechten Zwickel auf Griechisch: „Auferstehung“5 . In jedem Feld ist neben die Buchstaben ein Efeublatt mit Stängel gemeißelt.

\footnotetext{
1 Sandstein; Höhe: 155 , Breite: $89 \mathrm{~cm}$.

2 Ursprünglich war das Band erhaben ausgeführt; bei der Wiederherstellung des Steins nach dem Zweiten Weltkrieg (siehe Anm. 8) wurde es in die Schriftebene vertieft eingemeiBelt. Auch in seiner ersten Ausführung schnitt es in die Profilierung des Rechteckrahmens und des Bogens ein.

${ }^{3}$ ANN(O) / CHR(ISTI). M.D.I. I SIBI ET PO/STERITAT/I CAPNION/IAE IOANN/ ES REVCHL/IN PHORC/ENSIS . S - Das $S$ am Ende der Inschrift gilt allgemein als Abkürzung von $S A C R V M$ (geweiht, gewidmet); vgl. z. B.: Walther Ludwig, Nachlese zur Biographie und Genealogie von Johannes Reuchlin, in: Walther Ludwig, Miscella Neolatina. Ausgewählte Aufsätze 1989-2003, Bd.3 (Noctes Neolatinae, Bd.2.3), Hildesheim 2005, S.32-40, hier S.33. - Zu „Capnio“: Reuchlin benutzte häufig diese gräzisierte Namensform.

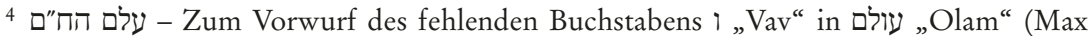
BRoD, Johannes Reuchlin und sein Kampf. Eine historische Monographie, Stuttgart 1965, S.342; Leiva Petersen, Goldstadt - ein Epitaph Reuchlins? - ad vicanos port ... [Vortrag am 19. April 1986], in: Pforzheimer Reuchlinpreis 1955-2005. Die Reden der Preisträger, 3., erweiterte u. ergänzte Aufl. [Supplemente zu den Schriften der Heidelberger Akademie der Wissenschaften, Philosophisch-historische Klasse, Bd.18], Heidelberg 2007, S.217-242, hier S.221; Franz Posset, Johann Reuchlin [1455-1522]. A Theological Biography, Berlin/ Boston 2015, S.215) siehe: David H. Price, Johannes Reuchlin and the Campaign to Destroy Jewish Books, Oxford 2011, S. 303 Anm.6.
}

5 ANA $\Sigma$ TA $\Sigma I \Sigma$. 


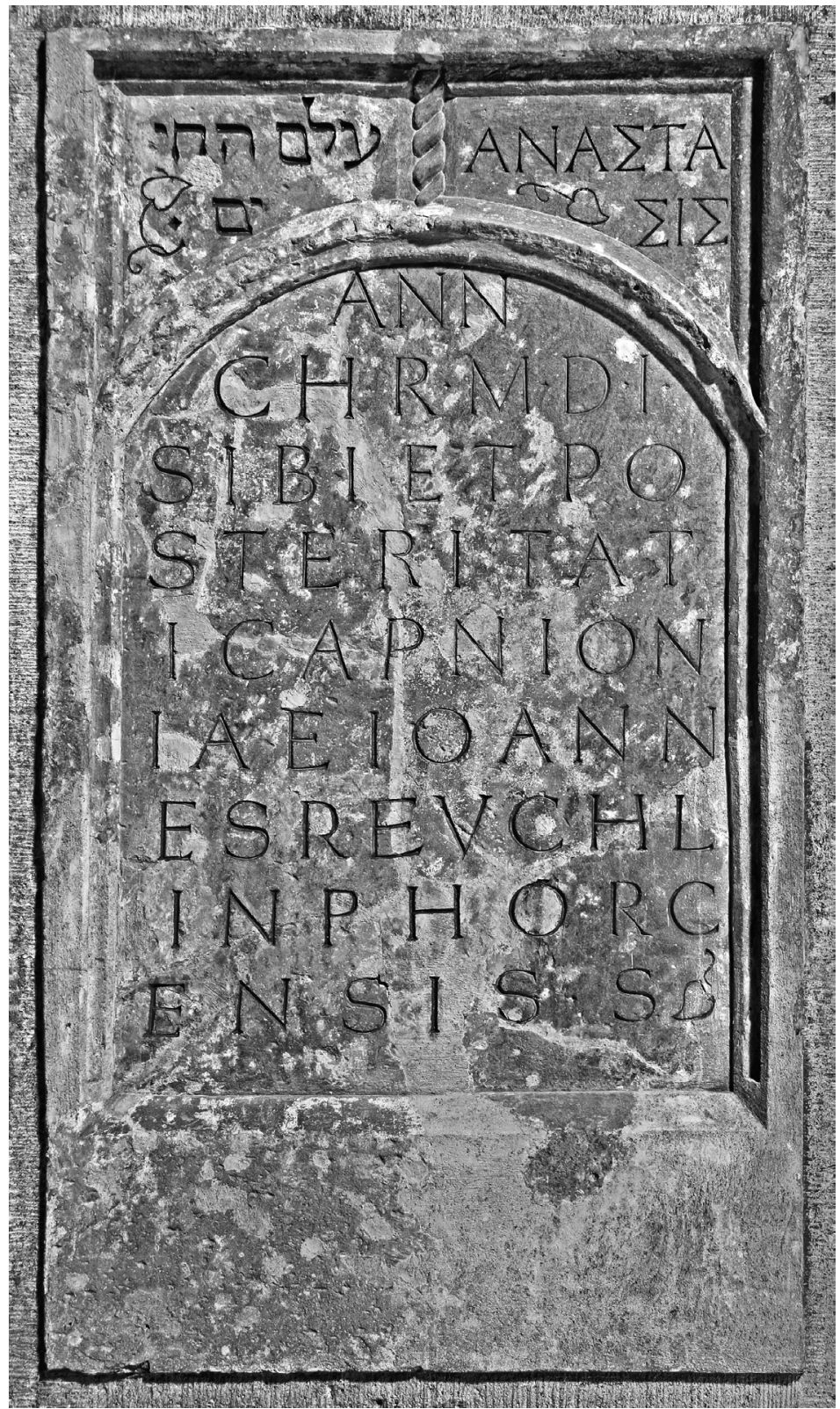

Abb.1: Reuchlin-Gedenkstein von 1501, seit 1955 in der Stuttgarter Leonhardskirche. 
Bei der lateinischen Inschrift orientieren sich Buchstabenform und Schriftbild an römischen Vorbildern. Alle Buchstaben wahren annähernd den gleichen weiten Abstand zueinander, selbst zwischen den einzelnen Worten gibt es keinen größeren Zwischenraum, und am Ende der Zeile wird auf Worte oder Silben ebenfalls keine Rücksicht genommen, der Umbruch erfolgt, wie es gerade ausgeht, ohne ein Trennungszeichen. Auch die - hier dekorativ eingesetzten - Efeublätter sind von der antiken Epigraphik übernommen, wo sie als Satzzeichen (hedera distinguens) gebraucht werden. Quadrangel als Trennzeichen finden sich im Hauptfeld nur bei den Zahlzeichen in der zweiten Zeile und vor $\operatorname{dem} S$ am Textende sowie - umwunden von dem Efeustängel - in der hebräischen Inschrift im linken Zwickel.

Mit Sicherheit geht nicht nur der Text der Inschrift, sondern die Gestaltung der Schrift und wohl auch des Steins insgesamt auf Reuchlin zurück. Denn der außergewöhnliche Stein folgt keinem gängigen Muster, und die Kenntnis antiker Denkmäler ist bei dem ausführenden Steinmetzen nicht zu erwarten.

Reuchlin - 1455 in Pforzheim geboren - hat die Tafel 1501, also im Alter von 45 Jahren, im Kreuzgang des Stuttgarter Dominikanerklosters aufstellen lassen. Ein Fantasie und Wirklichkeit vermischender Holzstich des Architekten Carl Friedrich Beisbarth (1808-1878) von 1855 (Abb.2) zeigt den Westflügel des Kreuzgangs mit Blick in Richtung Nordflügel, wo der Stein neben der Ecke zu erkennen ist $^{6} .1871$ hat man die Schriftplatte in ein Rahmenwerk mit Bildnismedaillon eingefügt. Derart zu einem Denkmal erweitert, erhielt der Stein einen neuen Platz an der nördlichen Außenwand der Kirche ${ }^{7}$. Dort, im Binnenhof des Kreuzgangs, an der Stelle des schon zuvor abgerissenen Südflügels, blieb er, bis er nach dem Zweiten Weltkrieg aus den Trümmern geborgen, wieder zusammengesetzt, zunächst ins Städtische Lapidarium gelangte und schließlich 1955 im Chor der Leonhardskirche aufgestellt wurde ${ }^{8}$.

${ }^{6}$ Holzstich in: Karl Alexander Heideloff (Hg.), Die Kunst des Mittelalters in Schwaben. Denkmäler der Baukunst, Bildnerei und Malerei, Stuttgart 1855, S. 31 Fig. 18. - Für seine ständige Anwesenheit im Kreuzgang sprechen die Erwähnungen in den Jahren 1624 (Johann Jakob Gabelkofer, Geschichte der Stadt Stuttgart, 1624. Mschr. Abschrift der Hs. Nr. 31 im StadtA Stuttgart von Paul NëGele, Stuttgart 1942, S.19f. [StadtA Stuttgart: Kc 548]), 1752 (Christian Friedrich SATTLER, Historische Beschreibung des Herzogthums Würtemberg, Stuttgart/Eßlingen 1752, Teil 1, S.34f. mit Abb.) und 1839 (Schwäbischer Merkur [Chronik], 14.10.1839, S.1121).

7 J[ulius] Hartmann, Chronik der Stuttgarter Hospitalkirche, Stuttgart 1888, S.22. Ansicht des Kreuzgangbinnenhofs mit dem Reuchlin-Denkmal: Gustav WaIs, Alt-Stuttgarts Bauten im Bild, Stuttgart 1951, Nr.52 (S.71). - Fotografie des Reuchlin-Denkmals: Paul Sauer, 500 Jahre Hospitalkirche (Veröffentlichungen des Archivs der Stadt Stuttgart, Bd. 62), Stuttgart 1993, Abb. 19 (S.30).

${ }^{8}$ Gustav WaIs, Die St. Leonhardskirche und die Hospitalkirche zu Stuttgart. Eine Darstellung der beiden gotischen Kirchen mit baugeschichtlichen und kunstgeschichtlichen Erläuterungen, Stuttgart 1956, S. 31 f. Nr. 34. - Die Bruchstücke des Reuchlin-Gedenksteins wurden nach ihrer Bergung 1949 von dem Bildhauer Willy Schönfeld zusammengesetzt. 


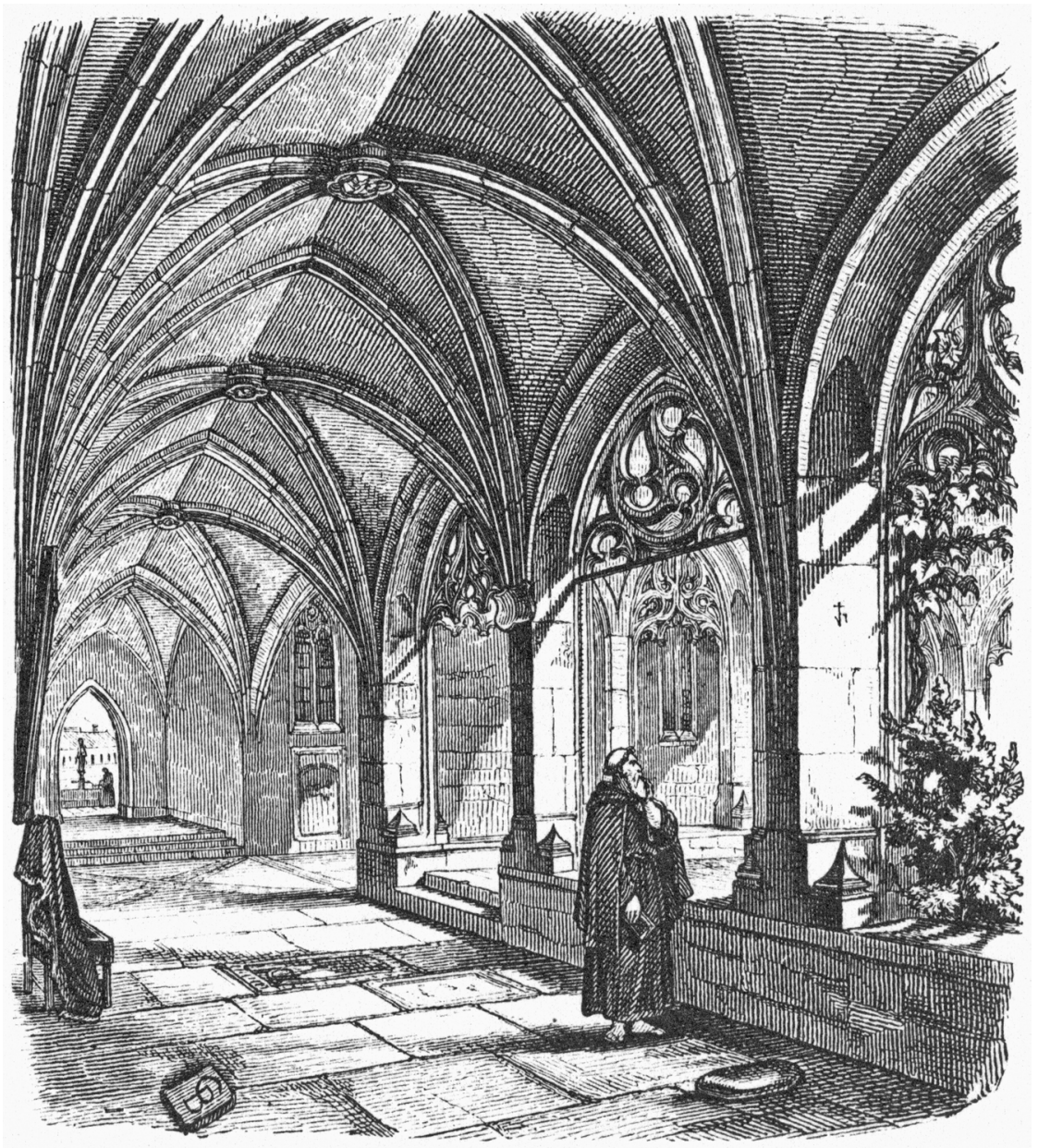

Abb. 2: Kreuzgang des Stuttgarter Dominikanerklosters, Holzstich von Carl Friedrich Beisbarth, 1855:

Wiedergegeben ist der Westflügel mit Blick in Richtung Nordflügel, wo der Reuchlin-Gedenkstein gleich neben der Ecke an der Wand angebracht ist. 
Reuchlin, dem 1481 eine Stelle an der jungen Tübinger Universität übertragen worden war', begleitete im folgenden Jahr seinen Landesherrn Eberhard im Bart nach Rom ${ }^{10}$ und hatte anschließend in dessen Diensten unter anderem verschiedene juristische Ämter inne ${ }^{11}$. Seinen Wohnsitz nahm er in Stuttgart. Um 1484 heiratete er eine Schwester des Stuttgarter Bürgers Hans Müller von Ditzingen ${ }^{12}$. Und 1498 erwarb er ein an die Nordseite der Stiftskirche grenzendes Haus ${ }^{13}$. In seinen sprachwissenschaftlichen Studien widmete er sich neben der griechischen besonders der hebräischen Sprache. Als in Köln der vom jüdischen Glauben zum Christentum konvertierte Johannes Pfefferkorn forderte, alle hebräischen Schriften, mit Ausnahme des Alten Testaments, zu verbrennen, und dabei die Unterstützung der Kölner Dominikaner erhielt, setzte sich Reuchlin gegen dieses Vorhaben heftig zur Wehr ${ }^{14}$. Die langwierige Auseinandersetzung mit den Dominikanern, die letztendlich 1520 von Papst Leo X. zugunsten der Gegenpartei entschieden wurde, führte offenbar dazu, dass Reuchlin sich nicht in der Dominikaner-, sondern in der Leonhardskirche bestatten ließ, wo sich auch das Grab seiner Frau befand ${ }^{15}$. Ob es sich

9 Sönke Lorenz, Reuchlin und die Universität Tübingen, in: Johannes Reuchlin und der „Judenbücherstreit“, hg. von Sönke Lorenz † und Dieter MerTens (Tübinger Bausteine zur Landesgeschichte, Bd. 22), Ostfildern 2013, S.15-53, besonders S. 16-20.

10 Lorenz (wie Anm. 9) S.22.

11 Reuchlin als Jurist: Adolf Laufs, Johannes Reuchlin. Jurist in einer Zeitenwende, in: Stefan RheIn (Hg.), Reuchlin und die politischen Kräfte seiner Zeit (Pforzheimer Reuchlinschriften, Bd. 5), Sigmaringen 1998, S.9-30; Dieter Stievermann, Johannes Reuchlin als Jurist und Rat in württembergischen Diensten, in: ebd., S.31-51; Horst CARL, Triumvir Sueviae. Reuchlin als Bundesrichter, in: ebd., S.65-86; Wolfgang SCHILD, Reuchlin als Jurist, in: Lorenz/Mertens (wie Anm. 9) S. 147-172.

12 Hansmartin Decker-Hauff, Bausteine zur Reuchlin-Biographie, in: Hermann KLING / Stefan RheIN (Hg.), Johannes Reuchlin (1455-1522), Nachdruck der 1955 von Manfred Kress hg. Festgabe, neu hg. u. erweitert (Pforzheimer Reuchlinschriften, Bd. 4), Sigmaringen 1994, S. 83-107, hier S. 86-90; Ludwig (wie Anm. 3) S. 37 Nr. 6; Günther Schweizer, Die Familie Reuchlin. Eine genealogische Bestandsaufnahme, in: Lorenz/Mertens (wie Anm. 9) S.223-262, hier S. 229.

13 Wais (wie Anm.7) Nr.7 (S. 22).

14 Kurze Zusammenfassung von Reuchlins Leben und Kampf gegen die Vernichtung hebräischer Schriften: Stefan RHEIn, Johannes Reuchlin (1455-1522). Ein deutscher „uomo universale“, in: Humanismus im deutschen Südwesten. Biographische Profile, im Auftrag der Stiftung „Humanismus heute“ des Landes Baden-Württemberg hg. von Paul Gerhard Sснмidt, Sigmaringen 1993, S.59-75. - Zu verschiedenen Aspekten seines Lebens und seiner Tätigkeiten siehe: Lorenz/Mertens (wie Anm. 9). - Ausführlich über den „Judenbücherstreit“: PrICE (wie Anm.4).

15 Diese Begründung findet sich in: Petrus Apianus und Bartholomeus Amantius, Inscriptiones sacrosanctae vetustatis, non illae quidem romanae, sed totius fere orbis, Ingolstadt 1534, S. CCCCLIX, wo es heißt: Hic lapis Stutgardiae apud fratres praedicatores visitur, Caeterum orta perfidia illorum apud D. Leonhardum in Suburbio ibidem cum vxore sepeliri voluit Anno Do. M.D.XXII. Mense Innio Die XXX. - Ferner in: Bonifatius AmerBACH, um 1544 (Ludwig Geiger [Hg.], Johannes Reuchlins Briefwechsel [Bibliothek des Litterarischen Vereins in Stuttgart, CXXVI], Tübingen 1875, S. 363 f.; Stefan RHEIN, Reuch- 
dabei um die zweite Ehefrau Reuchlins handelt, wie Haller vermutet und DeckerHauff für sicher hält ${ }^{16}$, muss letztlich offen bleiben, denn wir erfahren darüber nur: cum vxore sepeliri voluit ${ }^{17}$. Den Text seines Epitaphs in der Leonhardskirche, in dem Reuchlin - nach Humanistenart - überschwänglich gerühmt wird, verfasste sein Freund und Kollege, der in Cannstatt geborene Humanist Johann Alexander Brassicanus (1500-1539) ${ }^{18}$.

Reuchlin gilt neben Erasmus von Rotterdam (1466/67-1536) als der bedeutendste Humanist nördlich der Alpen. Seine wichtigste wissenschaftliche Leistung war die Schaffung der Grundlage für die christliche Hebraistik. Sein Nachruhm beruht aber vor allem auf seiner Haltung im Judenbücherstreit.

Schon seine Zeitgenossen betrachteten Reuchlin als herausragenden Gelehrten. So zollte ihm 1506 der Humanist Jakob Wimpfeling (1450-1528) höchste Anerkennung: „Johannes Reuchlin, in drei Sprachen hochgelehrt, von dem wir nicht wissen, ob wir ihn für den größeren Gräzisten oder Hebraisten, den größeren Philosophen, Theologen oder Juristen halten sollen: so sehr zeigt er Exzellenz in all diesen Wissenschaften. “19

liniana II. Forschungen zum Werk Johannes Reuchlins, in: KLING/Rhein [wie Anm.12] S.285-301, hier S.285). - Sowie in: Sammlung von Inschriften des 16. Jahrhunderts, um 1559/1560 (Handschrift), fol.16v (WLB: Cod. hist. qt. 58), (dazu: Johannes RaU, Über eine Sammlung von Inschriften des 16. Jahrhunderts, in: ZWLG 23 [1964] S. 418-438, hier S. 431 f., Nr. 38). - Und in: Gabelkofer (wie Anm.6) S. 19 f.

16 Johannes Haller, Die Anfänge der Universität Tübingen 1477-1537. Zur Feier des 450jährigen Bestehens der Universität, 2. Teil: Nachweise und Erläuterungen, Stuttgart 1929, S. 94\%; Decker-Hauff (wie Anm. 12) S. 98-101, zur zweiten Ehe: S. 90-93. - Ludwig (wie Anm.3) S.37f. Nr.7, bezeichnet Decker-Hauffs Angaben zur zweiten Ehe als problematisch; dabei bezieht er sich insbesondere auf dessen Argumentation, bei Reuchlins zweiter Frau handle es sich um Anna Decker. - Den einzigen spärlichen Hinweis auf eine zweite Ehe gibt Reuchlin in drei Briefen, indem er sich als digamus (zweimal verheiratet) bezeichnet (Johannes Reuchlin. Briefwechsel 1-4, bearb. von Matthias DALL'AsTA und Gerald DöRnER, hg. von der Heidelberger Akademie der Wissenschaften in Zusammenarbeit mit der Stadt Pforzheim, Stuttgart-Bad Cannstatt 1999-2013; hier: Briefwechsel 2, 2003, Nr. 190 Z. 41, Nr.191 Z. 91; Briefwechsel 4, 2013, Nr.349 Z. 23 u. 30).

17 Vgl. Anm. 15.

18 Den Text des verlorenen Epitaphs überliefern: Martin Crusius, Annales suevici, Bd.3, Frankfurt 1596, S. 576f.; GabelKofer (wie Anm. 6) S. 72 f.; Johannes Schmid, Inscriptiones monumentorum, quae sunt Stutgardiae. ... collectae et conscriptae per M. Johannem Schmid, Marppachensem, p.t. Stutgardiae ad d. Leonhardum pastorem, Anno MDCXXXX (Handschrift), S.304 (WLB Cod. hist. oct. 18). Letzterer teilt außerdem mit, es handle sich um ein „E[pitaphium]. Ein kleines Täfelin vf papir geschriben an der Saulen.“ Womit vermutlich der erste nördliche Arkadenpfeiler von Ost gemeint ist, an dem er noch drei weitere Epitaphe aufführt. Vgl. dazu: Ludwig (wie Anm.3) S.33-36 Nr.3.

19 Brief an Johannes Prüss, in: Jakob Wimpfeling. Briefwechsel, 2. Teilbd., eingeleitet, kommentiert u. hg. von Otto Herding und Dieter Mertens (Jacobi Wimpfelingi opera selecta, III/2), München 1990, Nr. 203 (S. 534). - Übersetzung ins Deutsche von Ulrich Gaier, in: Ulrich GAIER / Wolfgang SCHÜRLE (Hg.), Schwabenspiegel. Literatur vom Neckar zum Bodensee 1000-1800, Lesebuch 1: Suevia, Hof, Kloster, [Eggingen] 2004, S. 39. 
Wie stand Reuchlin zu Luther und zur Reformation? ${ }^{20} 1518$ hatte er seinem jungen Verwandten und Schüler Philipp Melanchthon (1497-1560) ${ }^{21}$ den Lehrstuhl für Griechisch an der Universität in Wittenberg vermittelt. Dort wurde Melanchthon schon bald zu einem Vertrauten und Freund Luthers und überredete ihn dazu, Reuchlin zu schreiben. In dem Brief bekundet Luther, dass er Reuchlins Bücher im Herzen trage, und er bezeichnet sich als seinen Nachfolger, denn nun schnappten die Zähne des höllischen Papsttums nach ihm, um irgendwie den Verlust an Ansehen auszugleichen, den sie durch Reuchlin (im Judenbücherstreit) erlitten hätten ${ }^{22}$. Eine Antwort Reuchlins ist nicht bekannt. Doch durch einen Brief Ulrichs von Hutten (1488-1523) aus dem Jahr 1521 erhalten wir Kenntnis davon, dass Reuchlin, um sich gegen die Anschuldigung des Papstes zu verteidigen, vorbrachte, er habe Luthers Sache immer missbilligt, und er habe Luther sehr Übel genommen, dass sich sein Name in dessen Schriften finde, und er habe sogar versucht, Luther die Anhänger abspenstig zu machen ${ }^{23}$. Dessen ungeachtet zeigt der Titelholzschnitt einer im selben Jahr (1521) in Straßburg gedruckten Schrift ${ }^{24}$ Reuchlin, Hutten und Luther, tituliert als „patroni libertatis“, denen zwei Gruppen von Luthergegnern gegenübergestellt sind. Damals wurden die drei „Anwälte der Freiheit“ von der Öffentlichkeit als Gemeinschaft wahrgenommen, die gemeinsam für die „freie Meinungsäusserung gegenüber inquisitorischer Verketzerungssucht“ eintritt ${ }^{25}$. Aber trotz aller Kritik an den kirchlichen Missständen lag es Reuchlin fern, gegen die Kirche und den Papst aufzubegehren oder sie gar in Frage $\mathrm{zu}$ stellen. Im Gegenteil dokumentiert der Eintrag seines Namens im Bruderschaftsbuch der Stuttgarter Salve-Regina-Bruderschaft unter den sacerdotes, dass er sich in seinen letzten Lebensjahren noch zum Priester hat weihen lassen ${ }^{26}$.

Reuchlins dreisprachiger Stein im Dominikanerkreuzgang fand früh Beachtung: Bereits 1534 wurde er erstmals in einer Inschriftensammlung publiziert ${ }^{27}$. Auf korrekte Maßverhältnisse hat man dabei offenbar keinen Wert gelegt (Abb.3).

20 Siehe dazu: Barbara Mahlmann-Bauer, Johannes Reuchlin und die Reformation Eine neue Würdigung, in: Reuchlins Freunde und Gegner. Kommunikative Konstellationen eines frühneuzeitlichen Medienereignisses, hg. von Wilhelm KüHLmann (Pforzheimer Reuchlinschriften, Bd.12), Ostfildern 2010, S.155-191.

${ }^{21}$ Zum Verwandtschaftsverhältnis zwischen Reuchlin und Melanchthon: ScHweizer (wie Anm.12) S. 230-232.

22 Johannes Reuchlin. Briefwechsel 4, Leseausgabe in deutscher Übersetzung von Georg BurKard, Stuttgart-Bad Cannstatt 2011, Nr. 352 (14.12.1518).

23 Ebd. Nr. 395 (22.02.1521).

${ }^{24}$ History Von den fier ketzren Prediger ordens, von Thomas Murner, Straßburg 1521. - Abb. in: Lorenz/Mertens (wie Anm. 9) S.121.

${ }^{25}$ Diese Formulierung verwendet Geiger in Bezug auf den Judenbücherstreit (GeIger [wie Anm.15] S.240).

${ }^{26}$ Ludwig (wie Anm.3) S.36f. Nr.5.

27 Apianus/Amantius (wie Anm. 15) S. CCCCLIX. - Aus dem Nachlass des Sohnes von Petrus Apianus blieb ein Band der „Insciptiones“ mit kolorierten Federzeichnungen erhalten (Frankfurt a.M., Universitätsbibliothek: Ms.lat.qu.102), der einen Teil der Inschriften 


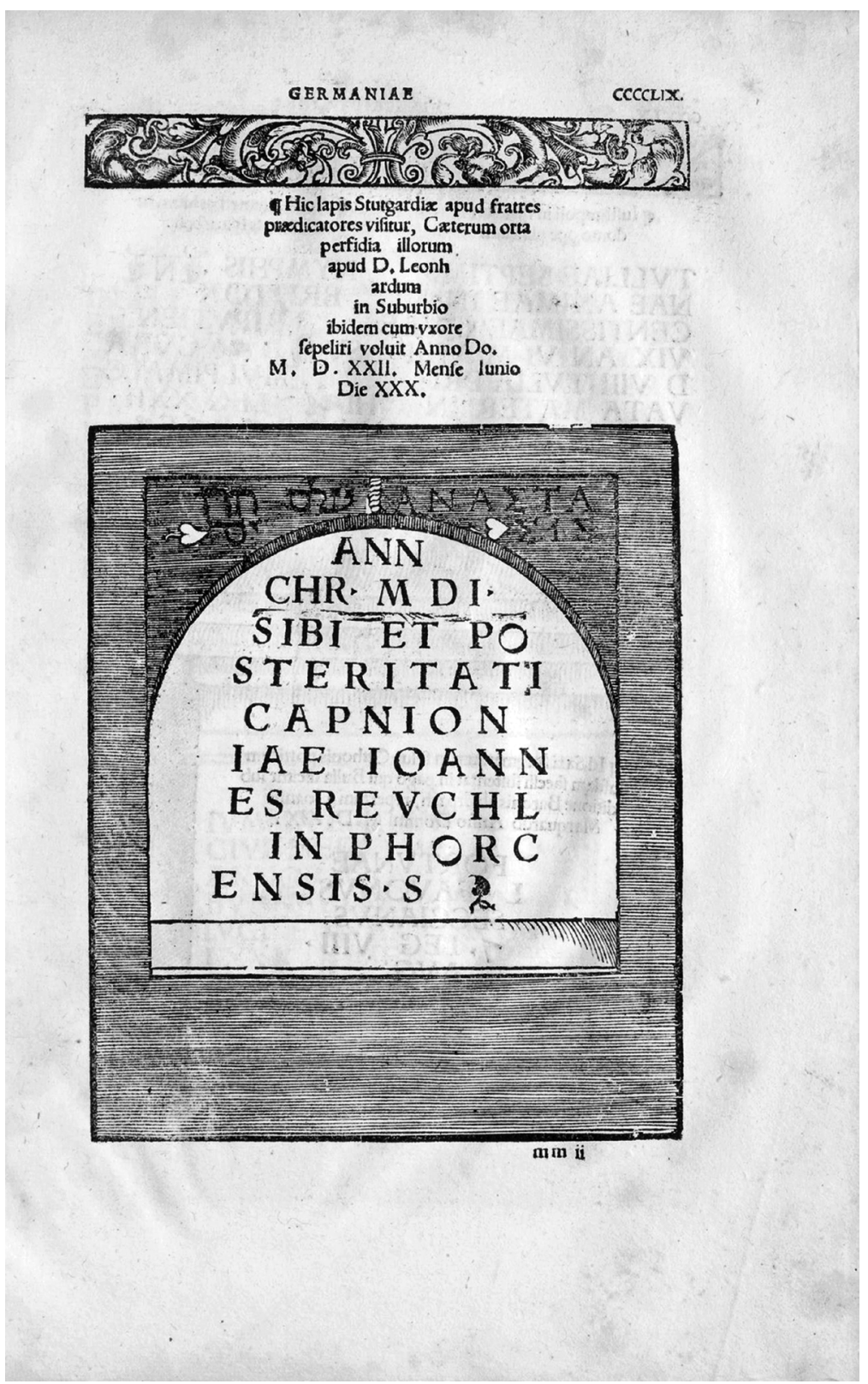

Abb. 3: Reuchlin-Gedenkstein in der von Apianus und Amantius publizierten Inschriftensammlung („Inscriptiones“), 1534. 
Welche Funktion war diesem seltsamen Stein von seinem Donator, Reuchlin, zugedacht? In der Literatur gilt er als Grabplatte, Grabstein, Grabdenkmal, Epitaph oder Gedenkstein ${ }^{28}$. Laut Decker-Hauff handelt es sich um ein Familienepitaph, das Reuchlin anlässlich des Todes seiner ersten Frau $^{29}$ habe errichten lassen ${ }^{30}$. Dazu passt allerdings nicht, dass die Inschrift nur von Reuchlin und seinen Nachkommen spricht. Seine Frau schließt das nicht mit ein, und Kinder hatten sie keine. Trotzdem ist zu vermuten, dass vor dieser an der Wand des Kreuzgangs angebrachten Tafel die Grabstätten des Humanisten und seiner Nachkommen vorgesehen waren. Zumindest die Zwickeltexte „Auferstehung“ und „Ewiges Leben“ weisen auf die Zeit nach dem Tod hin. Die in den Kreuzgangboden einzulassenden Gräber hätten dann Grabplatten mit den Namen und Sterbedaten erhalten. Der Stein würde also den Ort markieren, wo Reuchlin für sich und seine Nachkommen eine Grabstelle gestiftet hat. Den Bezug zu den Dominikanern hat Reuchlin übrigens gleichsam geerbt, denn sein Vater war weltlicher Verwalter des Pforzheimer Dominikanerklosters ${ }^{31}$.

Eine ganz andere These für die ursprüngliche Bestimmung des Steins stellte 1986 Leiva Petersen auf: Bei der in lateinischer Sprache abgefassten selbstbewussten Widmung an die Reuchlinsche Nachkommenschaft oder Nachwelt handle es sich um eine Bauinschrift, die Reuchlin an seinem 1498 neu erworbenen Wohnhaus habe anbringen lassen. Der „Aufsatz, der die Bogenplatte zu einer rechteckigen macht", also die Partie mit der hebräischen und griechischen Inschrift oberhalb des Bogens, sei später hinzugefügt worden. Auf diese Weise habe man zu unbekannter Zeit die ursprüngliche Bauinschrift in ein Grabdenkmal umgestaltet ${ }^{32}$.

des 1534 gedruckten, vorgenannten Werkes umfasst und als Vorarbeit zu diesem wesentlich umfangreicheren Werk zu betrachten ist. Auf fol.26v ist der Reuchlin-Gedenkstein abgebildet (Abb.4); dabei fällt auf, dass manche Zeilenumbrüche der lateinischen Inschrift vom Original (und somit auch von der 1534 gedruckten Darstellung) abweichen. Zum Frankfurter Manuskript: Gerhardt Powitz/Jutta Hager, Die neueren Handschriften der Gruppe Manuscripta Latina, Teil I: Allgemeiner Bestand (Kataloge der Stadt- und Universitätsbibliothek Frankfurt am Main, Bd. 6), Frankfurt a.M. 1988, S. 25 f.

28 Auch wenn sie die Problematik nicht eigens ansprechen, bleiben viele Autoren unentschieden, zum Beispiel: Wars (wie Anm. 8) S. 32 Nr.34: Grabmal, Denkmal, Grabstein, Grabplatte; Harald MöHring, Ev. St. Leonhardskirche Stuttgart (Schnell \& Steiner, Kunstführer Nr.1505), München/Zürich 1984, S.11: Gelehrtenepitaph, Gedenkstein; SaUER (wie Anm.7) S. 25: Grab- oder Gedenkplatte.

${ }^{29}$ Das Todesjahr seiner ersten Frau, einer Schwester des Stuttgarter Bürgers Hans Müller von Ditzingen, die er um 1484 geheiratet hat, ist nicht bekannt. Literaturangaben zu Reuchlins erster Ehe siehe Anm.12.

30 Decker-Hauff (wie Anm.12) S. 100.

31 Rhein (wie Anm. 14) S. 60.

32 Petersen (wie Anm. 4) S. 220-226, besonders S. 221, 225, 226 und 233. - Zu der Möglichkeit, dass es ursprünglich eine Hausinschrift war, neigt auch: Posset (wie Anm. 4) S. 215. 
[Die Abbildung kann aus rechtlichen Gründen online nicht bereitgestellt werden.]

Abb.4: Reuchlin-Gedenkstein, kolorierte Federzeichnung in einer Handschrift der „Inscriptiones“ aus dem Nachlass von Apianus, vor 1534 (UB Frankfurt a. M., Ms.lat.qu.102, fol.26v). 
Doch die Fakten sprechen gegen diese These: Zum einen besteht die Platte aus einem Stein, woran nachträglich nichts angestückt wurde. Und ohne die von Anfang an geplanten Inschriften in den Zwickeln wäre die Platte mit Sicherheit anders gestaltet worden, denn derart große, leere Zwickelfelder machen keinen Sinn. Zum anderen hätte die Platte, die sich 1534 nachweislich im Dominikanerkloster befand, bald nach Reuchlins Tod von der Hausfassade entfernt, durch das Hinzufügen der Zwickelinschriften in ein Gedächtnismal umgewandelt und in das noch bestehende Kloster transferiert werden müssen, in dem Reuchlin gar nicht bestattet worden ist. Wer hätte das veranlassen sollen, und aus welchem Grund? ${ }^{33}$

Seine Berühmtheit verdankt der Stein nicht zuletzt den Inschriften in drei Sprachen. Doch das war damals keine Ausnahme. Seit den Brüdern van Eyck wurde der Kreuztitulus bei anspruchsvollen Kreuzigungsdarstellungen nicht selten so wiedergegeben ${ }^{34}$, wie das Johannes-Evangelium berichtet: „Pilatus ließ ein Schild schreiben und es am Kreuz anbringen, darauf stand Jesus der Nasoräer, Judenkönig in hebräischer, lateinischer und griechischer Sprache. " ${ }^{35}$ Im gleichen Jahr als Reuchlin seinen Gedenkstein im Kreuzgang des Dominikanerklosters aufstellen ließ (1501), wurde für den Leonhardskirchhof ein von Hans Seyfer geschaffener Kreuzberg gestiftet ${ }^{36}$, dessen Kreuz eine solche dreisprachige Tafel erhielt (Abb.5). Deren Text ist scheinbar auf ein mehrfach gefaltetes Blatt geschrieben, das mit zahlreichen Nägelchen an eine Tafel geheftet wurde. Die Tafel ihrerseits ist oberhalb von Christi Haupt an den Kreuzesstamm genagelt. Wegen der Heiligkeit des Kreuzes sind die drei Sprachen als linguae sanctae angesehen worden. In der Bildhauerkunst des mittleren Neckargebietes finden sich die drei „heiligen Sprachen" noch in einem anderen Zusammenhang: Vier der Wasserspeier am Westturm der Heilbronner Kilianskirche stellen die Symbolwesen der Evangelisten dar, deren

33 Ludwig (wie Anm.3) S. 33 Nr.2, hält Petersens These ebenfalls für abwegig, da der Wortlaut, der manchmal falsch verstanden worden sei, eine Hausinschrift ausschließe. Seine Übertragung der lateinischen Inschrift ins Deutsche lautet: „Im Jahre Christi 1501 hat Johannes Reuchlin aus Pforzheim für sich und für die Capnionische [d. h. seine Reuchlinische] Nachkommenschaft dieses Monument geweiht“. Petersen hingegen vertritt die Ansicht, mit der Formulierung posteritati capnioniae seien nicht die leiblichen Nachkommen, sondern die Nachwelt, der Nachruhm Reuchlins gemeint (Petersen [wie Anm. 4] S. 224).

${ }^{34} \mathrm{Zu}$ den dreisprachigen Kreuztituli in der Kunst: Mechtild Онммаснт, Das Kruzifix des Niclaus Gerhaert von Leyden in Baden-Baden von 1467. Typus - Stil, Herkunft - Nachfolge (Europäische Hochschulschriften, Reihe XXVIII: Kunstgeschichte, Bd. 2), Bern/ Frankfurt a. M. 1973, S. $26 \mathrm{f}$.

35 Joh. 19,19-20; Übersetzung nach: Das Neue Testament und frühchristliche Schriften, übersetzt und kommentiert von Klaus Berger und Christiane Nord, Frankfurt a.M./ Leipzig 1999.

36 Zum Kreuzberg vom Stuttgarter Leonhardskirchhof: Heribert Meurer, Die Stuttgarter Kreuzigungsgruppe, in: Andreas Pfeiffer / Karl Halbauer (Hg.), Hans Seyfer. Bildhauer an Neckar und Rhein um 1500 (Heilbronner Museumskatalog, Bd. 105), Heilbronn 2002, S. 74-81 und Kat.-Nr.3 (S.130-132). 


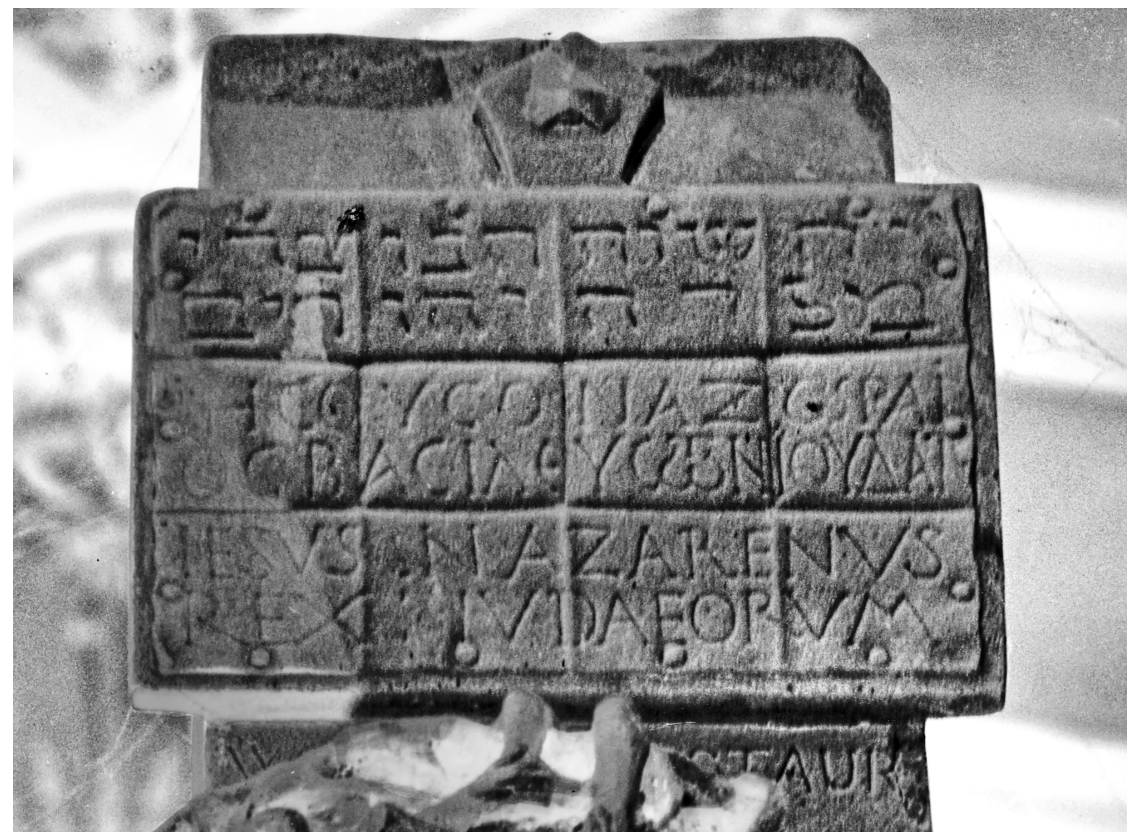

Abb. 5: Kreuztitulus der Kreuzigungsgruppe von Hans Seyfer, 1501; ursprünglich auf dem Stuttgarter Leonhardskirchhof, heute in der Hospitalkirche (ehem. Dominikanerkirche).

aufgeschlagene Bücher ursprünglich Bibelzitate in den drei Sprachen wiedergaben $^{37}$.

Fazit: Der Reuchlin-Gedenkstein war mit allergrößter Wahrscheinlichkeit dafür bestimmt, die Stelle zu kennzeichnen, an der Reuchlin für sich und seine Nachkommen einen Begräbnisplatz im Kreuzgang des Stuttgarter Dominikanerklosters gestiftet hat. Da sich Reuchlin dann aber in der Leonhardskirche bestatten ließ, der Stein jedoch bis nach dem Zweiten Weltkrieg im Dominikanerkreuzgang blieb, stand er letztendlich mit der tatsächlichen Grabstätte Reuchlins in keinem Zusammenhang.

37 Karl Halbauer, Der Westturm der Heilbronner Kilianskirche. Beschreibung und kunstgeschichtliche Einordnung, in: heilbronnica 3, 2006 (Quellen und Forschungen zur Geschichte der Stadt Heilbronn, Bd.17; Jahrbuch für schwäbisch-fränkische Geschichte, Bd. 35), S. 49-118, hier S. 74 mit Anm. 38, S.104f. mit Abb. 71. 